\title{
Airborne bacterial species in indoor air and association with physical factors
}

\author{
Anne Mette Madsen* and Afnan Barooni \\ National Research Centre for the Working Environment, Lersø Parkallé 105, 2100 Copenhagen Ø, Denmark
}

\begin{abstract}
The aim of this study is to obtain knowledge about which bacterial species are present in indoor air in homes, and whether physical factors affect the concentration and species composition of bacteria in indoor air. Measurements have been performed for one whole year inside five homes and once in 54 homes. The most species rich genera were Bacillus, Paenibacillus, and Kocuria. Among the gram-negative bacteria Paracoccus yeei was found frequently, and the concentration of this bacterium was associated significantly with season. The concentrations of gram-positive and gram-negative bacteria and species compositions were significantly associated with season. The concentrations of Kocuria palustris, Kocuria rhizophila, and Bacillus pumilus were associated positively or negatively with either absolute or relative humidity.
\end{abstract}

Peer-review under the responsibility of the organizing committee of the ICMB21.

Keywords: bacterial species; bioaerosols; Kocuria rhizophila; Paracoccus yeei, seasonality; ventilation.

\section{Introduction}

Airborne bacteria in indoor environments are causative agents to various infectious diseases and may be involved in either an increase or decrease in the risk of developing asthma and atopy. Studies of bacterial taxa in homes have mainly focused on floor, bed, or surface bacteria. However, air is an important transmission route for bacteria. Potential health risks of bacteria is usually evaluated at species level, and Matrix-assisted Laser Desorption Time of Flight (MALDI-TOF MS) has been revealed as a reliable and useful method for identification of bacteria from the indoor environment. Using that method for identification, it has been shown that the concentration of indoor Staphylococcus is associated with the indoor air change rate (ACR) and area per occupant indicating that it might be possible to affect the presence of Staphylococcus in indoor air [1]. In contrast relative humidity $(\mathrm{RH})$ of indoor air was not associated significantly with concentration of viable bacteria in general [2] and with three common genera [1].

The aim of this study is to obtain knowledge about which non-Staphylococcus bacterial species are present in indoor air in in Danish homes, and whether factors as season, ACR, RH, and absolute humidity (AH) affect the concentration as well as species composition of indoor air bacteria.

\section{Methodology}

The study has been performed with measurements for 1 whole year in living rooms in five homes without water damages; we used samples and data on ACR, RH, and temperature from our previous studies [1] [2]. To see whether the species found throughout one year in the five homes are representative we also analysed samples taken once in 54 homes. In the five homes air samples were taken using a GSP mounted with polycarbonate filters $(n=20)$, and it sampled airborne bacteria for $6 \mathrm{~h}$ during each measurement once each season. In all homes samples were also taken using the Electrostatic dust collector (EDC, $n=74)$ which was placed on an open surface allowing dust to settle on a cloth for 1 month. The dust sampled on the filters and the cloths was extracted and following plated on nutrient agar plates and incubated at $25^{\circ} \mathrm{C}$. All bacterial colonies were counted and identified using MALDI-TOF MS. Data on species other than Staphylococcus are part of this study. Statistical analysis were performed as mixed model with random effect of home.

\section{Results}

\section{Bacterial species}

In the five homes some species were found frequently, but in low concentrations, e.g. Moraxella osloensis, while other species e.g. Bacillus megaterium and Paenibacillus glucanolyticus were found seldom but in high concentrations. The gram-negative bacterium Paracoccus yeei was observed repeatedly, and it constituted a large part of the airborne gram-negative bacteria (Fig. 


\section{ICMB21}

1a). In the five homes, eleven different Bacillus species were found (Fig. 1b). Of gram-positive bacteria other than Bacillus species the species Kocuria rhizophila was very commonly found and in high concentrations (Fig. 1c).
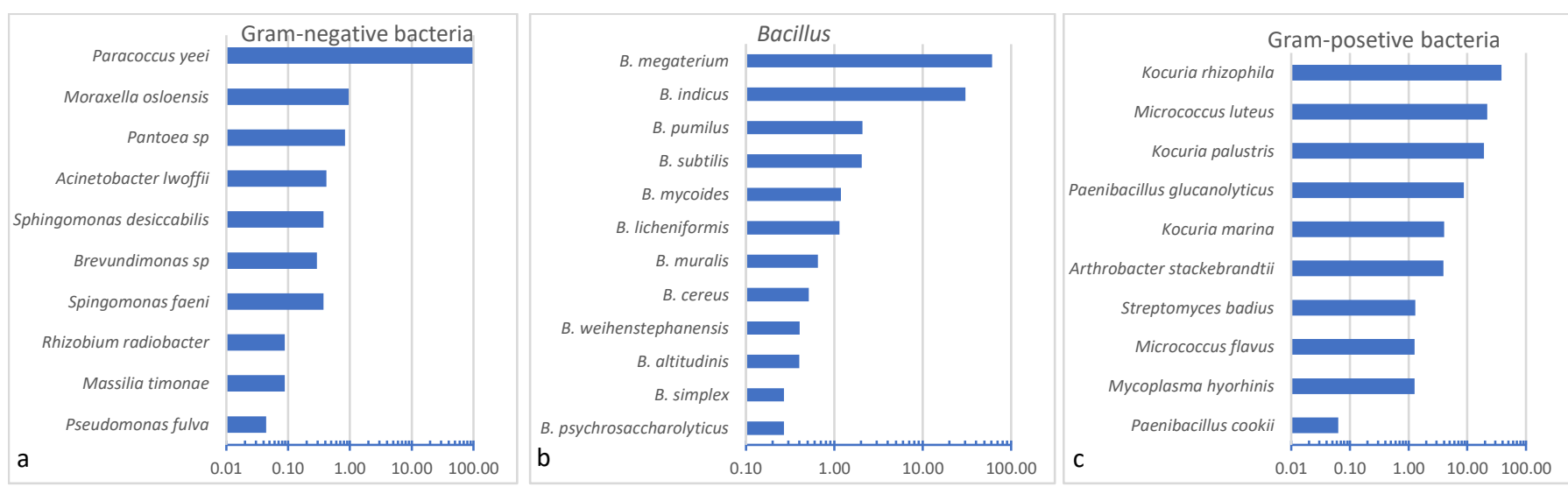

Fig. 1 abc. Top ten gram-negative bacteria (a), the eleven Bacillus species (b), and top ten gram-positive bacteria other than Bacillus and Staphylococcus (c) found in five homes throughout a year; all presented as $\%$ of total isolates within the category.

In the 54 homes the gram-negative bacteria found in highest concentrations and most frequently were Moraxella osloensis, Paracoccus yeei, Acinetobacter lwoffii, and Pseudomonas oryzihabitans. For the genus Bacillus this was: Bacillus pumilus, Bacillus cereus, Bacillus licheniformis, and Bacillus megaterium, and for the other gram-positive bacteria: Micrococcus luteus, Kocuria palustris, Kocuria rhizophila, and Micrococcus terreus.

\section{Bacteria as affected by physical factors}

The concentration of gram-negative bacteria as measured using the GSP samplers was affected by season $(p=0.0016)$ but was not associated with RH, AH, and ACR. The highest concentrations were found in autumn and spring and the lowest in summer. Concentrations of Paracoccus yeei were associated negatively with indoor temperature $(\beta=-7.86, p=0.046)$, and was also affected by season $(p=0.0086)$ with highest concentrations in spring. For gram-positive bacteria sampled with GSP, a significant effect of season $(\mathrm{p}=0.0024)$ was found with highest concentrations in autumn and spring. Micrococcus flavus was associated negatively with ACR $(\beta=-1.22, p=0.015)$ and Bacillus pumilus positively with $\mathrm{RH}(\beta=3.86, p=0.048)$. Kocuria rhizophila was associated positively with $\mathrm{RH}(\beta=7.4, p=0.022)$ and negatively with temperature $(\beta=-7.4, \mathrm{p}=0.050)$.

In the cross-sectional study the concentration of gram-negative bacteria as measured using the EDC sampler was not associated with RH, AH, ACR, and season. For gram-positive bacteria sampled with the EDC significant effects of AH $(\beta=-6.2, p=0.016)$ and season $(p=0.0004)$ were found. Higher concentrations were found in spring than in summer $(p=0.019)$ and winter $(p=0.027)$. The concentration of Kocuria palustris was associated negatively with $\mathrm{AH}(\beta=-7.70, p=0.040)$. Kocuria rhizophila was associated negatively with temperature $(\beta=-10.0, p=0.031)$. Redundancy analysis (RDA) show that the bacterial species composition can be separated significantly by season.

\section{Discussion}

In this study, we show that in addition to the previously found Staphylococcus species [1] other bacterial species are found frequently in indoor air in homes. For some species, associations between concentrations and seasons were found.

Some of the found species are described as opportunistic pathogens, e.g. Moraxella osloensis, some as common in soil e.g. Bacillus licheniformis, one as protective against allergy: Acinetobacter lwoffii, some as part of the normal microbiota of the upper airways e.g. Rothia amarae or skin e.g. Roseomonas mucosa, and for some species very little is known, e.g. Lysinibacillus fusiformis and Arthrobacter stackebrandtii. Within the same genus different relations to the physical environment were found, e.g. Kocuria rhizophila was associated positively with $\mathrm{RH}$ and Kocuria palustris negatively with $\mathrm{AH}$, and this highlights the importance of discriminating between species within the same genus. In this study we used MALDI-TOF MS for bacterial identification, and if we had used NGS we expected to have found fewer species but more genera.

\section{References}

1. Madsen, A.M., et al., Concentrations of Staphylococcus species in indoor air as associated with other bacteria, season, relative humidity, air change rate, and S. aureus-positive occupants. Environ Res, 2018. 160: p. 282-291.

2. Frankel, M., et al., Seasonal variation of indoor microbial exposures and their relations to temperature, relative humidity and air exchange rates. Applied and Environmental Microbiology, 2012. 78: p. 8289-8297. 